\title{
CXLIX. THE UROCARMINE REACTION.
}

\author{
BY WILLIAM ROBERT FEARON AND \\ ARTHUR GEOFFREY THOMPSON \\ (Rockefeller Foundation Research Fellow in Clinical Biochemistry). \\ From the Physiological Department, Trinity College, Dublin.
}

(Received July 25th, 1930.)

WHILE examining some miscellaneous urines collected for class work a specimen was observed to give a carmine colour on treatment with concentrated hydrochloric acid and an oxidising agent such as hydrogen peroxide or potassium chlorate. The reaction was at first ascribed to the presence of urorosein or the vague scatoxyl-reds, but the solution in amyl alcohol failed to show the absorption bands characteristic of these substances. The reaction was then investigated, and the conclusion was reached that the colour arose from a condensation between indoxyl and $o$ - or $m$-cresol present in the urine, in accordance with the equation:

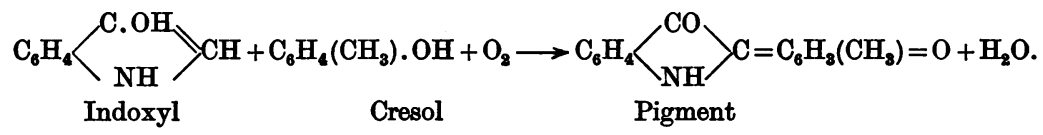

Since such a condensation may occur in any specimen of urine which contains $o$ - or $m$-phenolic compounds in addition to indoxyl, it serves to explain certain hitherto obscure colour reactions of urine, and is of importance in the estimation of indoxyl. In order to distinguish them from other red urinary pigments, such as indirubin, urorosein, and the scatole-reds, it is proposed to call these coloured condensation products urocarmine compounds, adopting a term originally used by Florence [1908] in a less definite sense.

Method of demonstrating the urocarmine test. This is a modification of the indigo-blue test done under conditions less favourable for the production of the blue dye; 2 cc. of urine are treated with two drops of $0.1 \%$ potassium chlorate solution or of $3 \%$ hydrogen peroxide, and then $10 \mathrm{cc}$. of colourless concentrated hydrochloric acid are added. Colourless hydrochloric acid, obtained by distillation, is used to avoid the variable oxidation factor due to the use of an acid which has turned yellow from the formation of oxides of chlorine. The mixture is well shaken, and after standing for a few minutes is extracted first with chloroform and then with amyl alcohol. Under these conditions most normal urines give a variable indigo-blue reaction, the pigment dissolving in the chloroform, but from time to time specimens will be found 
which display a reddish colour not removed by extraction with chloroform, but very soluble in amyl alcohol. With the specimen of urine under examination, an intense carmine colour developed at once and no indigo-blue could be detected in the mixture or in the chloroform extract.

Identification of the pigment. In order to determine if the chromogen of urocarmine were volatile in steam a litre of the urine was treated with a slight excess of $10 \%$ barium chloride and rapidly steam-distilled. During distillation the organic sulphates were decomposed by the barium, and the volatile phenols liberated. This method is more satisfactory than the usual one of acidifying the urine as it obviates darkening of the liquid and other changes. Distillation was continued until samples no longer gave a positive reaction with Millon's reagent. The residual urine was then concentrated to its original bulk on a water-bath, and filtered free from sulphate and carbonate. It was now found that the residue, after acidification, gave only a very faint urocarmine reaction but showed instead a definite indigo-blue. The distillate, after concentration, gave no colour of any kind on treatment with oxidising agent and acid, but a mixture of the concentrated distillate and the residual urine once more gave a urocarmine reaction. These facts suggested that two precursors were concerned in the formation of the colour, a non-volatile constituent, identified as indoxyl, and a volatile constituent, phenolic in character, which could not be identified with certainty by the paraformaldehyde test of Denigès [1913], but which was probably $o$ - or $m$-cresol, as well as ordinary phenol; $p$-cresol, the second principal urinary phenol, does not interact with indoxyl to yield a urocarmine.

The identity of the non-volatile constituent was established by obtaining a positive colour reaction with (1) a normal urine rich in indoxyl; (2) a solution of indoxyl prepared by the oxidation of indole by potassium persulphate; (3) a $1 \%$ solution of indoxyl synthesised from phenylglycine-o-carboxylic acid. The identity of the volatile constituent was confirmed by obtaining urocarmine pigments from o-cresol and indoxyl, and from phenol and indoxyl, indistinguishable from the pigment produced in the original urine, although it was not found possible to obtain this in a crystalline form on concentration of the amyl alcohol extract.

The condensation between phenols and indoxyl. Indoxyl readily undergoes an oxidative condensation with many phenols to produce leuco-bases and pigments. This is the reason why Molisch's carbohydrate test cannot be applied to urine, and why urines preserved with thymol develop a pink colour on treatment with strong acids. Two of these condensations, with $\alpha$-naphthol and thymol respectively, have been recognised and investigated by Jolles [1915], who has made them the basis of methods for detecting and estimating indoxyl. A colour reaction with resorcinol has been used in this department for some time as a class test for indoxyl. Following the study of the urocarmine reaction, the behaviour of phenols of biological interest towards indoxyl was investigated. Under the conditions of the reaction pigments were obtained with all phenolic compounds unsubstituted in the $p$-position, such 
as ordinary phenol, resorcinol, catechol, $o$ - and $m$-cresol, pyrogallol, orcinol, thymol, gallic acid, adrenaline, and $\alpha$-naphthol. Pigments could not be obtained, however, from the interaction between indoxyl and any of the $p$-substituted compounds examined, such as quinol, pure $p$-cresol, tyrosine, vanillin, homogentisic acid, and $\beta$-naphthol, which suggests that condensation takes place between the indoxyl and the phenol in the unsubstituted $p$-position of the latter to form the leuco-base. Indeed, the reaction might be of use in determining the presence or absence of $p$-substitution.

The constitution of the urocarmine pigments. From his study of the $\alpha$-naphthol and thymol condensation with indoxyl Jolles follows Friedländer in ascribing an indolignone structure to these bodies. The cresol derivative almost certainly belongs to the same type of compounds, having the structural formula:

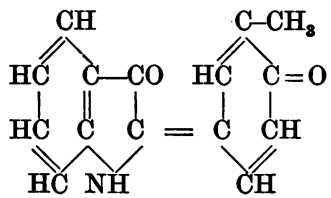

Hydrolysis of the pigment gave rise to anthranilic acid, due to rupture of the indole ring at the $\alpha$-carbon. The nature of the other fragment of the pigment molecule could not be ascertained, owing to lack of material. Until this has been accomplished it is not possible to locate the methyl group, but from analogy with the thymol compound, analysed by Jolles, it occupies the position indicated.

The urocarmine reaction in urine. When an indoxyl test is applied to urine in the ordinary way by adding an equal volume of concentrated hydrochloric acid of indefinite activity followed by an oxidising agent, most of the indoxyl present is rapidly converted into indigo-blue, and rendered inert, since in this form it can no longer interact with phenols to produce urocarmines. If however the test be modified as previously described, a variable amount of the indoxyl combines with the urinary phenols to form urocarmine, and escapes the transformation into indigo-blue. The extent of this formation of urocarmine depends on three factors: (1) the concentration of acid, and the temperature of the solution; (2) the concentration of $o$ - and $m$-phenols already present in the urine; (3) the nature of the oxidising agent employed. On the addition of resorcinol or $o$-cresol followed by acidification most, if not all, normal urines give a urocarmine reaction, and this may be made the basis of a simple colorimetric method for the estimation of indoxyl. The nature of the oxidising agent is important. Using hydrogen peroxide or potassium persulphate in the cold the colours obtained with resorcinol and o-cresol soon reach a maximum and remain unchanged for several hours. If however the solution be warmed, or if a stronger oxidising agent be employed, such as $1 \%$ potassium chlorate or hypochlorite, the colour deepens and the shade changes. In normal urine this was found to be due to a condensation between indoxyl and creatinine, and for this reason most urines containing indoxyl will give a 
urocarmine reaction if suitably oxidised, especially if the condensing acid be warm.

The indogenide reactions of urine. Those colour reactions of urine which are associated with indole comprise two groups: Urocyanine reactions [Heller, 1847], in which blue pigments are produced, and Urorubine reactions [Heller, 1847; Thudichum, 1867], in which red pigments are produced. These reactions have been abundantly investigated by Maillard [1913], who has traced the subject back to the seventeenth-century writings of Bellini, and who has summarised the observations of some hundreds of investigators as follows: "L'indigotine et l'hemi-indigotine suffisent donc à identifier toutes les couleurs bleues qui aient jamais été signalées dans l'urine humaine." This conclusion has not been affected by subsequent research, although Maillard's own conception of indigotine, or indigo-blue proper, as a simple polymer of hemi-indigotine does not appear to have attracted attention. (The term "hemiindigotine" is applied by Maillard to the blue indigoid pigment produced by the condensation of two molecules of indoxyl and extractable with chloroform.) Maillard's conclusion that all the urocyanine reactions are due to indigo formation has not been seriously challenged.

With regard to the Urorubine reactions of urine, however, considerable doubt still exists. The discovery of indirubine, or indigo-red, the red isomer of indigo-blue, suggested to Schunck [1857], Carter [1858], and Hoppe-Seyler [1863] that this might be the urorubine pigment of urine. Indirubine was actually isolated from urine, and identified by Rosin [1891], and its production was subsequently explained by Ellinger [1903, 1913] as being due to the formation of isatin by over-oxidation of indoxyl, and subsequent condensation of isatin and indoxyl to produce indigo-red. This simplified explanation has not been accepted by Maillard, who finds in the production of indirubine an explanation of most of the red pigments obtained from urine. A characteristic of indirubine is its solubility in chloroform, and Maillard has collected proof that all the chloroform-soluble red pigments obtained from urine by oxidation of the acidified urine are in reality one substance only-indigo-red. Hence it is possible to subdivide the urorubine reactions into two groups, (1) those producing pigments soluble in chloroform (e.g. indigo-red), and (2) those producing pigments insoluble in chloroform (e.g. urorosein, urocarmine). Pigments in the latter group are freely soluble in amyl alcohol, and may be termed the "alcohol-soluble" urorubines. The recognition of the urocarmines as a separate sub-group now renders it possible to simplify the classification of the urorubine reactions of urine as shown in Table I.

This classification does not extend to those miscellaneous red pigments observed from time to time, the descriptions of which are insufficiently definite to enable them to be identified. Among these are: uroerythrine [Simon, 1845], purpurine [Bird, 1846], uromelanine [Thudichum, 1867], the pigments of Rosenbach [1889], Baginsky [1892], Cotton [1902], Florence [1908], Arnold [1909], and many others up to the urorubine of Weiss [1930]. Some of these 


\section{Table I. Classification of the red indogenide pigments.}

(A) Pigments soluble in chloroform.

Indirubine or indigo-red.

(B) Pigments insoluble in chloroform.

1. Urocarmines. Simple condensation products of indoxyl and urinary phenols unsubstituted in the para-position. Both chromogen and pigment are soluble in amyl alcohol. Chromogen precursors can be resolved by steam distillation into volatile and non-volatile components.

(a) Phenol indogenide.

(b) Cresol indogenides.

The list might be extended to include the indogenides of the rarer urinary phenols, catechol and resorcinol, which are not volatile in steam.

2. Indole-reds. Produced by the autoxidation of indole. Only likely to be formed when indole occurs free in urine, a condition so rare as to be improbable.

(a) Indole-red. [Oddo, 1916; Baudisch and Hoschek, 1916.]

(b) Urorubin. [Weiss, 1930.]

3. Scatole-reds, or uroroseins. [Porcher and Hervieux, 1905.] Derived directly or indirectly from the biochemical oxidation of scatole or of tryptophan. Sometimes included among the indole-reds. Chromogen precursors cannot be resolved by steam distillation.

(a) Indolepropionic acid. [Stokvis, 1901.]

(b) Indoleacetic acid. Believed by Herter [1908] to be the chromogen of the original urorosein of Nencki and Sieber [1882].

(c) Indolealdehyde. Believed by Ellinger and Flamand [1909] to be the chief chromogen of the scatole-reds.

(d) Indolecarboxylic acid. [Maillard, 1913.]

In alcoholic solution the scatole-reds show well-defined absorption spectra.

have been identified by Maillard as indigo-red, but from the method of preparation it is probable that several of them are mixtures of various compounds, including the urocarmines. A careful study of the scatole- and indole-reds will almost certainly result in a simplification of the table.

Table II. Diagrammatic classification of the pigments.

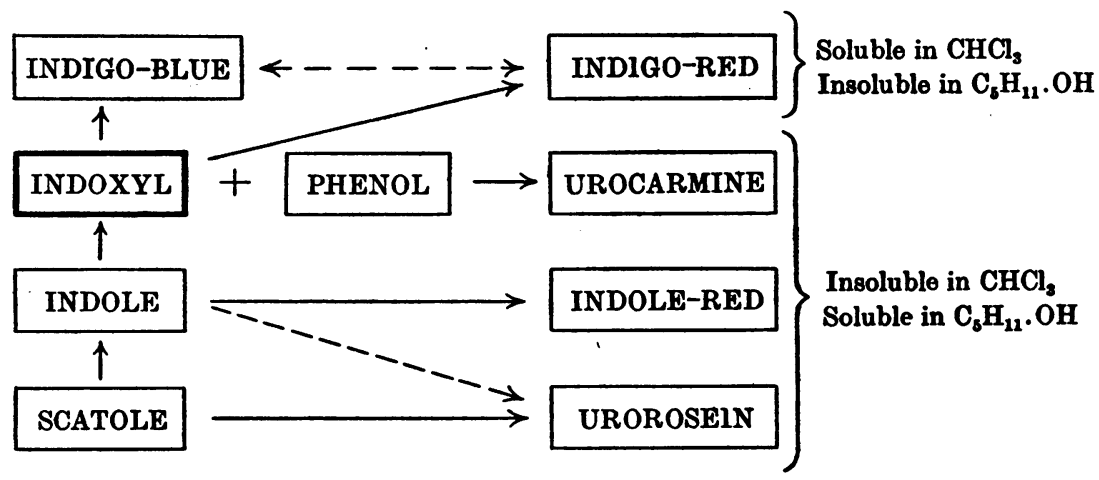

The urocarmine reaction with indole. Solutions of indole do not give a urocarmine reaction with the appropriate phenols, such as resorcinol, $o$-cresol, or thymol, unless the mixture is strongly oxidised (e.g. with potassium chlorate), when colours are obtained identical with the corresponding indoxyl colours. This is probably due to the oxidation of indole to indoxyl. Hence, by using $3 \%$ hydrogen peroxide and $1 \%$ potassium chlorate as oxidising agents in 
the cold, it is possible to distinguish between indole and indoxyl, since the former only gives the urocarmine reaction with the chlorate, whereas the latter gives a urocarmine with both oxidisers.

Significance of the urocarmine reaction in urine. When positive, this indicates the presence in the urine of indoxyl and phenols unsubstituted in the para-position, and hence constitutes a test for phenoluria. Its clinical interpretation has not yet been undertaken, but we observe that there appears to be no close association between the occurrence of indoxyl and of phenols in urine. A specimen may be abnormally rich in the indoxyl salt, and yet be quite free from phenols capable of giving a urocarmine reaction. The interesting problem of the relationship between para-cresol, the commonest substituted phenol in the urine, and the chromogenic phenols has also to be elucidated. Presumably the same mechanism by which homogentisic acid is produced from tyrosine is also concerned in the production of ortho- and meta-substitution compounds from a para-precursor.

\section{ExPERIMENTAL.}

Synthesis of typical urocarmine pigments.

Jolles [1915] having obtained condensation compounds of indoxyl with thymol and with $\alpha$-naphthol, it was decided to prepare the cresol and resorcinol compounds. As no suitable commercial source of indoxyl could be found this substance was synthesised from phenylglycine-o-carboxylic acid following the method of Macbeth and Craik [1925]. By this means a litre of $1.2 \%$ indoxyl in $25 \%$ acetic acid was obtained. This solution was used for the majority of the experiments.

o-Cresol indogenide. o-Cresol (1 g.) was dissolved in $100 \mathrm{cc}$ of $1.2 \%$ indoxyl solution, $20 \mathrm{cc}$. of pure concentrated hydrochloric acid were added, and the mixture was boiled gently on an electric hot plate under a reflux condenser for 2 hours. After standing overnight the mixture was slowly concentrated in a water-bath, with periods of cooling, until the excess of unchanged phenylglycine-o-carboxylic acid had separated and could be removed. The motherliquor was then diluted, treated with another $20 \mathrm{cc}$. of hydrochloric acid, and, the theoretical amount of $3 \%$ hydrogen peroxide having been added gradually, the concentration was continued. The autoxidation of the chromogen was hastened by the careful addition of $3 \%$ hydrogen peroxide solution. After standing overnight, the deeply coloured residue was dissolved in warm acetic acid, and twice recrystallised from this solvent. It was finally obtained as short needles, dark purple in colour, and with a metallic lustre.

M.P. $($ uncorrected $)=196-199^{\circ}$.

Analysis showed, Nitrogen (1) $5 \cdot 8 \%$;

Theoretical value $5 \cdot 97 \%$. 
Hydrolysis of the pigment by boiling in aqueous solution liberated anthranilic acid, which was identified as the ethyl ester. From this it is concluded that the body is a simple indogenide and is formed in accordance with the equations:

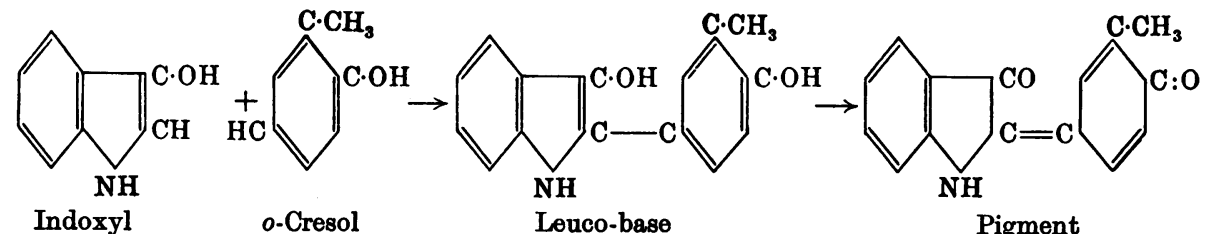

Resorcinol indogenide. This was prepared by a similar method, but the subsequent purification was complicated by the fact that a second chromogen was formed during the condensation. One of these chromogens gave a carmine pigment on oxidation, and resembled in many ways the cresol and thymol indogenides. The other chromogen gave a deep violet on oxidation in the presence of excess of acid. The carmine pigment was stable on dilution with water; the violet pigment was instantly decolorised, but was restored on again adding excess of acid. Both pigments were insoluble in chloroform, but soluble in amyl alcohol. It is concluded that they are the isomeric indogenides:<smiles>O=C1C(=O)C2C(=O)NC1C=CC2O</smiles>

Red<smiles>O=C1C(=O)C2C=CC1C(O)C2C(=O)Nc1ccccc1</smiles>

Violet

A fair yield of the labile violet pigment was obtained by boiling an excess (5 g.) of resorcinol with $100 \mathrm{cc}$. of $1.2 \%$ indoxyl in the presence of concentrated hydrochloric acid, under a reflux condenser in a current of coal gas for 6 hours. Subsequent oxidation with hydrogen peroxide produced only the violet pigment. Using resorcinol as a test for indoxyl, under ordinary conditions the pigment formed is almost entirely the red isomer.

\section{SUMmaRY.}

1. The term urocarmine has been applied to a class of indogenide pigments obtained from the condensation of indoxyl with various phenols unsubstituted in the para-position.

2. The preparation and isolation of $o$-cresol urocarmine is described.

3. The urocarmine reaction accounts for many of the pigments obtained from urine which are insoluble in chloroform.

4. A simple classification has been drawn up of the red pigments derived from urine. 


\section{REFERENCES.}

Arnold (1909). Z. physiol. Chem. 61, 240.

Baginsky (1892). Arch. Kinderheilk. 13, 312.

Baudisch and Hoschek (1916). Ber. deutsch. chem. Ges. 49, 2579.

Bird (1846). Urinary deposits (London, 2nd ed.).

Carter (1858). Edinburgh Med. J. 4, 85.

Cotton (1902). J. Pharm. Chim. 16, 258.

Denigès (1913). Chimie analytique, 4th ed., p. 212 (Paris).

Ellinger (1903). Z. physiol. Chem. 39, 44.

(1913). Analyse des Harnes (Huppert-Neuberg, Berlin). and Flamand (1909). Z. physiol. Chem. 62, 276.

Florence (1908). J. Pharm. Chim. 27, 145.

Heller (1847). Arch. Pharm. 98, 203.

Herter (1908). J. Biol. Chem. 4, 253.

Hoppe-Seyler (1863). Arch. pathol. Anat. 27, 388.

Jolles (1915). Z. physiol. Chem. 95, 29.

Macbeth and Craik (1925). Proc. Roy. Irish Acad. B 7, 37, 53.

Maillard (1913). Dictionnaire de physiologie (Richet, Paris).

Nencki and Sieber (1882). J. prakt. Chem. 26, 333.

Oddo (1916). Gazzetta, 46, 323.

Porcher and Hervieux (1905). J. Phys. Path. Gen. 7, 815.

Rosenbach (1889). Jahrsb. Thierchem. 19, 458.

Rosin (1891). Arch. path. Anat. 123, 519.

Schunck (1857). Phil. Mag. 14, 288.

Simon (1845). Animal chemistry (London).

Stokvis (1901). Jahrsb. Thierchem. 31, 444.

Thudichum (1867). J. Chem. Soc. 13, 339.

Weiss (1930). Klin. Wochenschr. 9, 248. 